\title{
Escaping from a chaotic saddle in the presence of noise
}

\author{
Rubén Capeáns, Juan Sabuco and \\ Miguel A.F. Sanjuán*
}

Nonlinear Dynamics, Chaos and Complex Systems Group, Departamento de Física, Universidad Rey Juan Carlos, Tulipán s/n, 28933 Móstoles, Madrid, Spain

Email: ruben.capeans@urjc.es

Email: juan.sabuco@urjc.es

Email: miguel.sanjuan@urjc.es

*Corresponding author

\begin{abstract}
There are certain situations in noisy nonlinear dynamical systems, for which it is required a fast transition between a chaotic and a periodic state. Here, we present a novel procedure to achieve this goal in the context of the partial control method of chaotic systems. The partial control method is a recently developed control procedure, that allows to keep the dynamics of a system showing transient chaos, close to its chaotic saddle. In this kind of systems, and in absence of an external control, trajectories remain chaotic for a while in a certain region of phase space before eventually escaping towards an external attractor. The aim of the control algorithm proposed here, is to maintain the chaotic orbits as much time as we want close to the chaotic saddle before forcing an immediate escape. To do that, we use the safe sets defined in the partial control method in a completely different way. By only using this set, we show how possible is to handle the stabilisation and destabilisation of the chaotic dynamics of the partially controlled system.
\end{abstract}

Keywords: chaos control; transient chaos; Lorenz system.

Reference to this paper should be made as follows: Capeáns, R., Sabuco, J. and Sanjuán, M.A.F. (2017) 'Escaping from a chaotic saddle in the presence of noise', Int. J. Nonlinear Dynamics and Control, Vol. 1, No. 1, pp.78-86.

Biographical notes: Rubén Capeáns received his BS in Physics from the University of Santiago de Compostela, Spain, in 2010. He is a graduate student working for his $\mathrm{PhD}$ in Physics in the field of controlling methods of chaotic dynamics at the University Rey Juan Carlos, Madrid, Spain.

Juan Sabuco received his BS in Telecommunications Engineering from the University Miguel Hernández, Elche, Spain, in 2008 and PhD in Physics from the University Rey Juan Carlos, Madrid, Spain, in 2013. His main field of research is control of chaotic dynamics. Currently, he is an Assistant Professor of Physics at the University Rey Juan Carlos.

Miguel A.F. Sanjuán received his BS in Physics from the University of Valladolid, Spain, in 1981, and PhD in Physics from the National University at a Distance, Madrid, Spain, in 1990. He is a Full Professor of Physics at the University Rey Juan Carlos, Madrid, Spain, where he is the Head of the 
Nonlinear Dynamics, Chaos and Complex Systems Group. He is Foreign Member of the Lithuanian Academy of Sciences and Corresponding Academician of the Spanish Royal Academy of Sciences.

\section{Introduction}

Chaotic trajectories have been traditionally seen as an undesirable behaviour which should be suppressed. However, in recent years, there have appeared different scenarios where the chaotic behaviour can be desirable. In mechanics, for example, chaos helps to prevent undesirable resonances (Schwartz and Triandaf, 1996). In engineering, the thermal pulse combustor is more efficient in the chaotic regime (In et al., 1997). In living organisms, chaotic dynamics in vital functions can make the difference between health and disease (Perc and Marhl, 2006). In biology, it has been suggested that the disappearance of chaos may be the signal of a pathological behaviour (Yang et al., 1995). In addition, there are systems where the transient dynamics evolves to an undesirable state like in Capeáns et al. (2014), where after a chaotic transient behaviour, one of the species gets extinct, or in the cancer model described in López et al. (2014), where the dynamics evolves towards an undesirable tumour growth.

Transient chaos is an unstable chaotic behaviour, in which the uncontrolled trajectories behave chaotic in a certain region of the phase space before eventually escaping to an external attractor. With the aim to maintain the transient chaotic behaviour indefinitely, in Sabuco et al. (2012a, 2012b), a robust strategy called partial control was proposed. The method is based on the use of certain sets in the phase space (Blanchini, 1999) and highlights for being able to handle noisy systems and the use of a smaller amount of control in comparison with the amount of disturbances affecting the system. To do that, the control method computes the set of points in the chaotic region of phase space, which require a small control to remain in. This set is called the safe set. The partial control method has been successfully applied to several paradigmatic systems like the Hénon map or the Duffing oscillator (Sabuco et al., 2012a), as well as other models in the context of ecology or cancer dynamics (Capeáns et al., 2014; López et al., 2014).

The aim of this work is not to maintain the transient chaotic behaviour forever, but only during certain period of time, after which the trajectories are forced to escape. See Figure 1. The easiest way of doing it would be first to apply the control, and stop it after the desirable period of time. As the dynamics is chaotic transient, the trajectories eventually will escape without external intervention. However, the time interval between the moment in which the control is stopped and the moment in which the trajectories leaves the chaotic region, can be very long in the presence of external disturbances. This time can vary widely depending on the parameters of the system, the initial conditions, or the random influence of disturbances. 
Figure 1 The goal of the control method (see online version for colours)
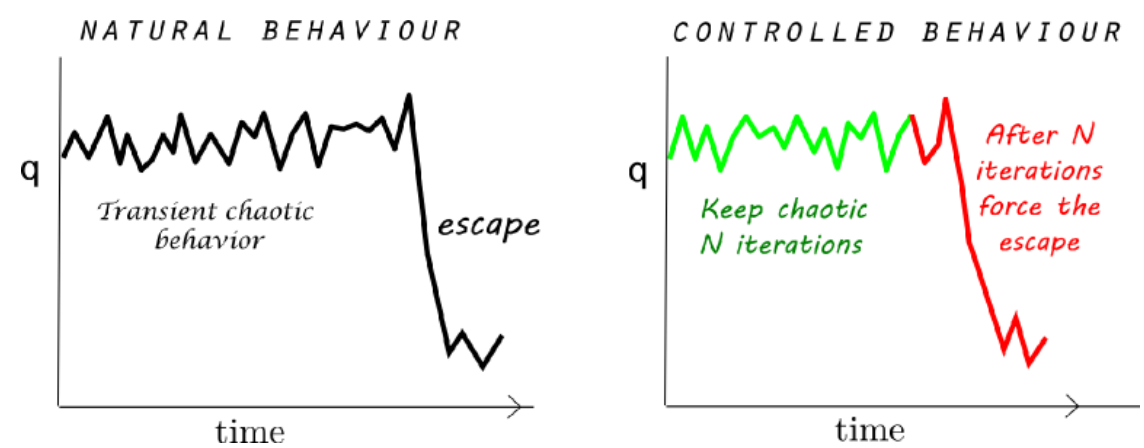

Notes: The figure on the left represents a transient chaotic orbit which eventually escapes (crisis) to another dynamical behaviour. The period time of the transient depends on the parameters of the model, the initial condition, and the random influence of the disturbances. The figure on the right represents the objective of the control strategy. It consists of sustaining the transient behaviour for $N$ iterations, where the choice of the $N$ value is totally arbitrary, and after that, to force the escape of the trajectory.

In this sense, we have found that it is possible to use the same safe set computed in the partial control method, to greatly reduce the escape time of the trajectories in the chaotic region. We show in this paper, how this is possible with the use of small perturbations and the safe set mentioned earlier, to stabilise (keep chaotic) or destabilise (fast escape) the transient chaotic dynamics. The structure of the paper is as follows. Section 2 is devoted to a description of the control method and its corresponding application to the logistic map and the Hénon map as paradigmatic models. Finally, some conclusions are drawn in Section 3.

\section{The control method}

In Sabuco et al. (2012a), the foundations of the partial control method are described. The method is applied on maps affected for some bounded disturbance $\xi_{0}$, so that the controlled dynamics is $q_{n+1}=f\left(q_{n}\right)+\xi_{n}+u_{n}$ where $u_{n}$ is the control applied. By a previous identification of the transient chaotic region in the phase space, an algorithm called sculpting algorithm (Sabuco et al., 2012a) is able to obtain a set of points (the safe set) in this region, for which the upper control value necessary to remain in this set is $u_{0}<\xi_{0}$. This counterintuitive result means that it is possible to keep the trajectories in the safe set, by using a control smaller than the disturbance affecting it. Notice that the control is applied to drive trajectories inside the safe set.

We face a completely different scenario if at some moment we want to force the trajectories to abandon the safe set and therefore escape to an external attractor. We show here that an optimal way to achieve this goal is to apply the control to drive the trajectories outside of the safe set. The simplest strategy is to apply the control $\left|u_{n}\right| \leq u_{0}$ each iteration to the most far away point in the map of the safe set. As we will show in the examples, this strategy reduces significantly the average time escape, in comparison with the average time escape if no perturbations are applied.

The procedure can be summarise in these steps: 
1 Given the map affected by a bounded disturbance $\xi_{0}$, identify the transient chaotic region that we call $Q_{0}$.

2 Take an upper value of control $u_{0}<\xi_{0}$, and apply the sculpting algorithm to obtain the safe set. Notice that the safe set is a subset of the region $Q_{0}$.

3 Keep the trajectories in the chaotic region: The control $\left|u_{n}\right| \leq u_{0}$ is chosen with the knowledge of $f\left(q_{n}\right)+\xi_{n}$, and applied to place the trajectory again in the set.

4 To force a fast escape of the trajectory from the chaotic region, the control $\left|u_{n}\right| \leq u_{0}$ is chosen with the knowledge of $f\left(q_{n}\right)+\xi_{n}$, and applied to place the trajectory in the furthest point respect to the safe set computed before.

In Figure 2, we can see a scheme representing the safe set acting in a dual way. In an attractive mode, when the trajectories are kept chaotic, and in a repulsive mode to speed up the escape of the trajectories. To show this procedure, two examples are drawn in the next section.

Figure 2 The two ways to apply the control in the safe set (see online version for colours)

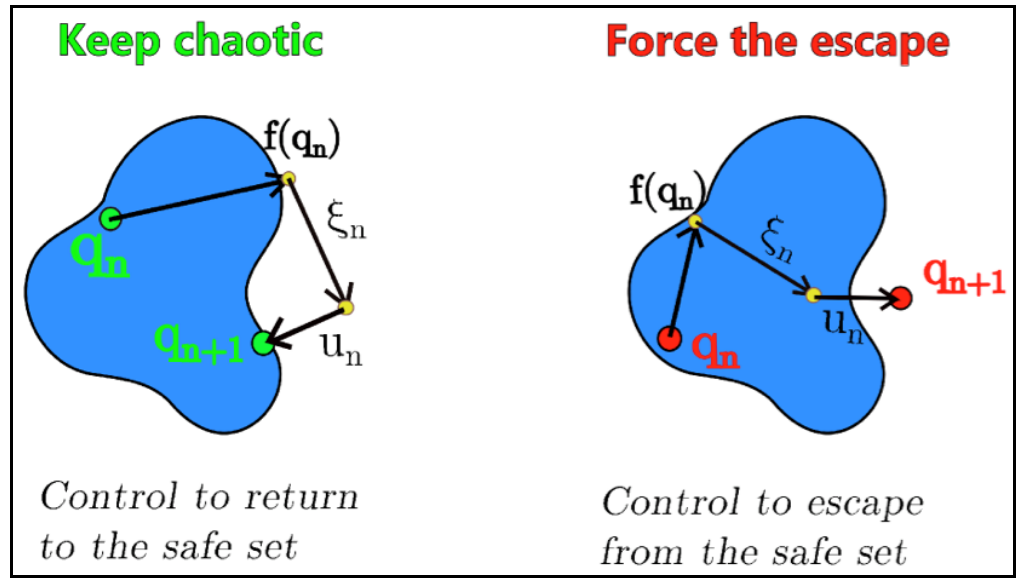

Notes: In this figure, we represent a scheme of the safe set and how the control is applied. We assume that some disturbance $\xi_{n}$ is affecting the dynamics, so that $q_{n+1}=f\left(q_{n}\right)+\xi_{n}+u_{n}$. In the figure on the left, the goal of the control $u_{n}$ is to go back to the safe set to keep the chaotic transient behaviour. In the figure on the right, the control is applied to push aside the orbit from the safe set and therefore produce the escape.

\subsection{The logistic map}

The logistic map is defined as follows:

$$
x_{n+1}=r x_{n}\left(1-x_{n}\right)
$$

where $x \in[0,1]$ and $r \in[0,4]$ to keep orbits in the interval $[0,1]$. Transient chaos appears in the logistic map for parameter values $r>4$. In order to compute an example, we have fixed $r=4.1$. For this value, the orbits starting in the interval $[0,1]$ typically abandon the interval after a long transient. In addition, we have considered that these 
orbits are affected by disturbances with a bound $\xi_{0}$. The effect of this disturbance can be both, to accelerate or to slow down the time escape depending on the particular contribution of the random disturbances in each iteration of the map. To keep the chaotic trajectories in the interval $x=[0,1]$, we consider to apply the control $u_{n}$ bounded by $u_{0}$. In this way, the dynamics of the partially controlled map is

$$
\begin{aligned}
& x_{n+1}=r x_{n}\left(1-x_{n}\right)+\xi_{n}+u_{n} \\
& \left|\xi_{n}\right| \leq \xi_{0}, \quad\left|u_{n}\right| \leq u_{0}
\end{aligned}
$$

Figure 3 Controlled dynamics in the logistic map, (a) and (b) natural escape (without forcing the escape) (c) and (d) applying control to force the escape (see online version for colours)

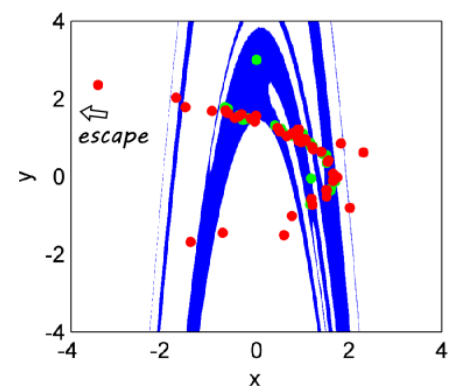

(a)

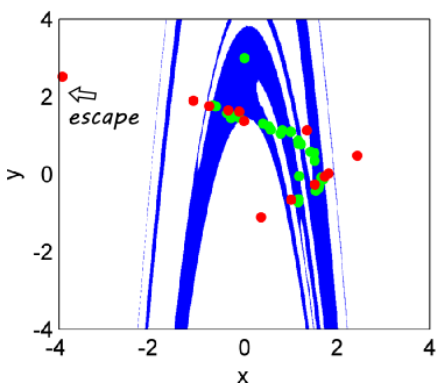

(c)

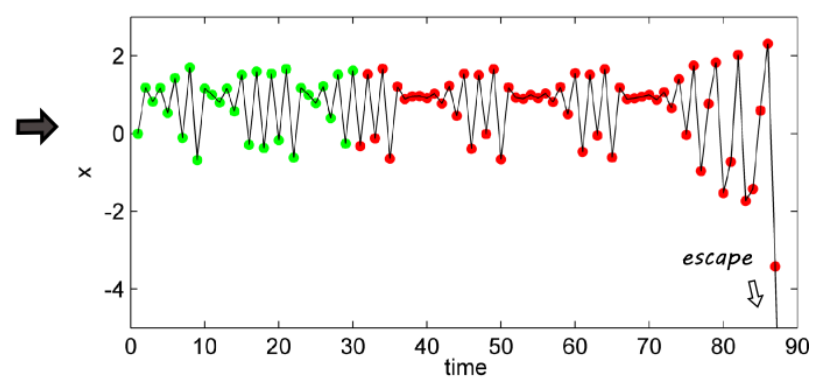

(b)

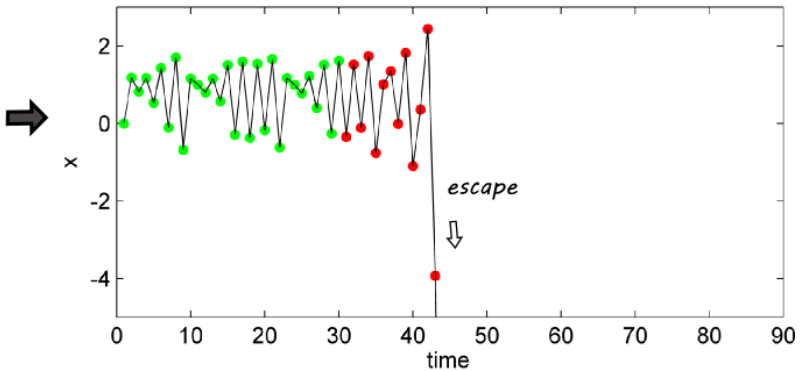

(d)

Notes: The black line in figures (a) and (c), represent the logistic map for the parameter $r=4.1$. For this value, transient chaos appears and orbits starting in the interval $[0,1]$ eventually escapes to $-\infty$. In order to apply the control, the safe set was computed for the value of disturbance $\xi_{0}=0.03$ and control value $u_{0}=0.02$. The safe set is showed with thick blue bars to help the visualisation. In the first 10 iterations (green points) the control is applied to return the orbit to the safe set. Then, in figure (a) the orbit is free to escape (no control is applied). However in figure (c) the orbit is forced to escape (red points). In figures (b) and (d) the corresponding time series are displayed. Notice that, by inducing the escape, the time to abandon the interval $[0,1]$ is greatly reduced.

For this example, we have chosen the values $\xi_{0}=0.03$ and $u_{0}=0.02$. Then, we compute the safe set showed in Figure 3. Imagine now that for instance, we want to keep the dynamics in the interval $[0,1]$ during ten iterations and then induce the escape. In Figures 3(a) and 3(b), we show the evolution of the variable $x$, where the control is applied in the first ten iterations to return orbits to the safe set. After that, we stop 
applying the control and the trajectory eventually escapes after a long time. In Figures 3(c) and 3(d), we represent the same situation with the difference that, after the first ten iterations, the control keeps applied with the goal of forcing its escape. As we can see in Figure 4, the average time escape is much smaller when the control is applied. In addition, the standard deviation of the time escape associated to the forced orbits is much smaller, which ensures that most orbits will escape very soon.

Figure 4 Average time escapes (see online version for colours)

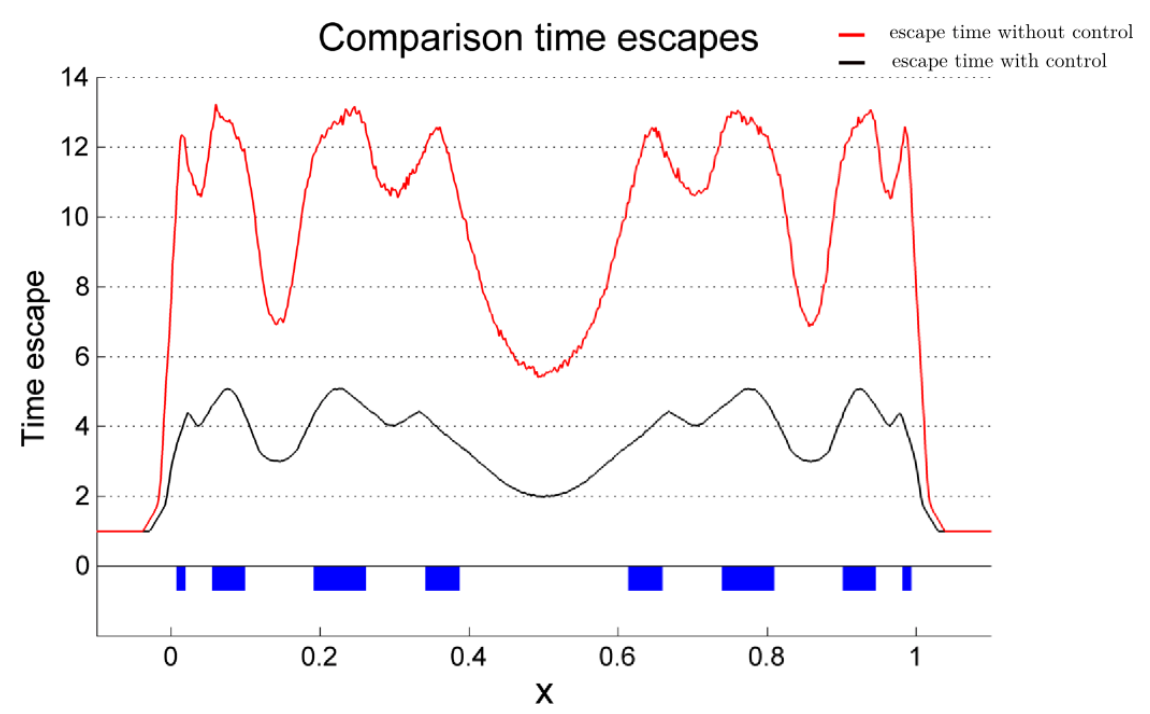

Notes: The figure represents the interval $[0,1]$ and the safe set (in blue) for the same conditions as the previous figure. The upper red line shows the average time escape when the orbit abandons the interval $[0,1]$ without the application of any perturbation. The lower black line shows the average time escape when the orbits are forced to escape by applying small controls. In this way, the trajectory escapes about 2.5 times faster than without control.

\subsection{The Hénon map}

The Hénon map is described by

$$
\begin{aligned}
& x_{n+1}=a-b y_{n}-x_{n}^{2} \\
& y_{n+1}=x_{n}
\end{aligned}
$$

Transient chaos appears in this system for a wide range of parameters $a$ and $b$. To show how the control method works in this system, we have taken $a=2.13$ and $b=0.3$. In addition, we consider that a bounded disturbance $\xi$ is affecting the variables $x$ and $y$. For this map, the trajectories starting in the square $x \in[-4,4]$ and $y \in[-4,4]$ have a chaotic transient characterised by a long escape time. After that, the trajectories escape out of this region toward infinity and never get back.

In order to keep trajectories in the square $x \in[-4,4]$ and $y \in[-4,4]$, we have applied a bounded control $u$ in the variables. The partially controlled dynamics can be rewritten as 


$$
\begin{aligned}
& x_{n+1}=a-b y_{n}-x_{n}^{2}+\xi_{i n}+u_{i n} \\
& y_{n+1}=x_{n}+\xi_{j n}+u_{j n} \\
& \sqrt{\left(\xi_{i n}\right)^{2}+\left(\xi_{j n}\right)^{2}} \leq \xi_{0} \quad \sqrt{\left(u_{i n}\right)^{2}+\left(u_{j n}\right)^{2}} \leq u_{0}
\end{aligned}
$$

Figure 5 Controlled dynamics in the logistic map, (a) and (b) natural escape (without forcing the escape) (c) and (d) applying control to force the escape (see online version for colours)

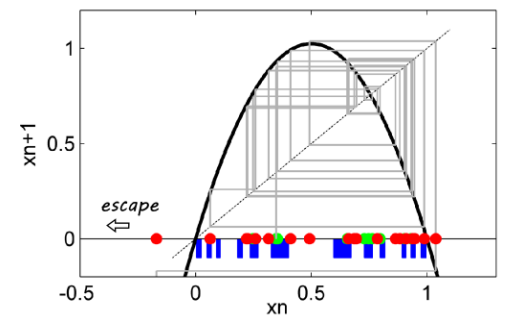

(a)

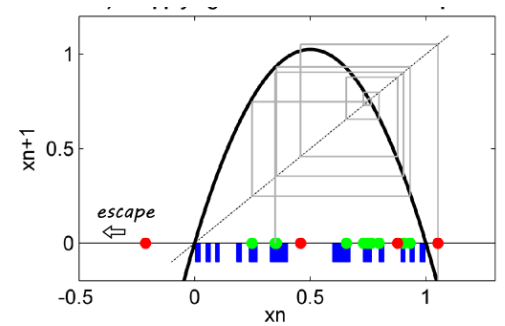

(c)

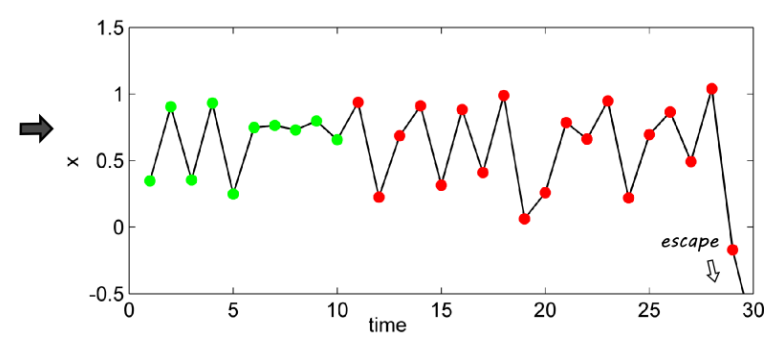

(b)

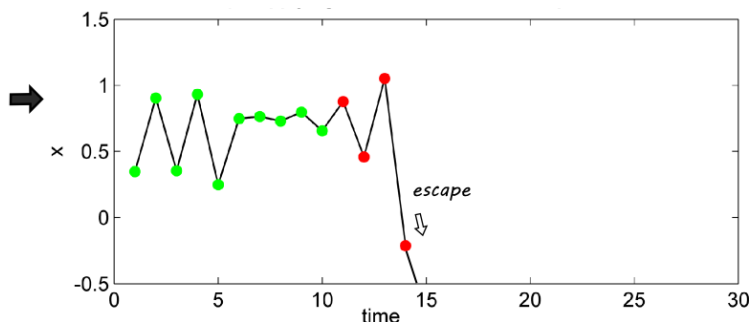

(d)

Notes: Figures (a) and (c), represent in blue the safe set computed for the Hénon map with the parameters $a=2.13$ and $b=0.3$, and for the value of disturbance $\xi_{0}=0.09$ and control $u_{0}=0.06$. The control is applied in two ways represented by green and red points. In the first 30 iterations (green points), the control drives the orbits to return to the safe set. In figure (a), after this first 30 iterations the applications of control is stopped, and the orbits are free to escape (red points). However, in figure (c), the control is applied to induce the escape and as a result the time escape is significantly reduced. In figures (b) and (d), the corresponding time series of the variable $x$ is showed.

In this case, we choose as an example the values $\xi_{0}=0.09$ and $u_{0}=0.06$ for the disturbances and control respectively. Next, we compute the safe set showed in Figure 5. In this case, we choose 30 iterations to keep the trajectory in the chaotic region, and after that, induce the escape. In Figure 5(a), we represent a trajectory in which the escape is not forced, while in Figure 5(c), the trajectory is forced to escape by the application of perturbations to move away of the safe set. As a result, the trajectory escapes in much less iterations [see the corresponding time series in Figures 5(b) and 5(d)]. To show a fair comparison of the time escape, we represent in Figure 6 the average time escape for both strategies, free escape and forced escape. The escape with the second strategy is about five times faster which supposes a big improvement, and all of that by using $\left|u_{n}\right| \leq u_{0}<\xi_{0}$ at each iteration. 
Figure 6 Average time escapes, (a) average time escape (without forcing the escape) (b) average time escape forcing the escape (see online version for colours)

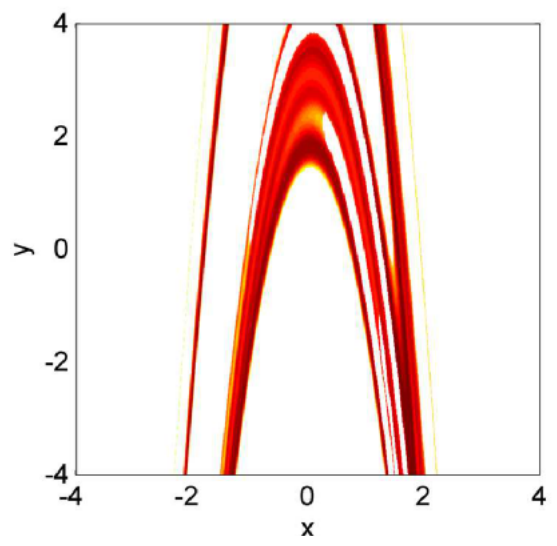

(a)

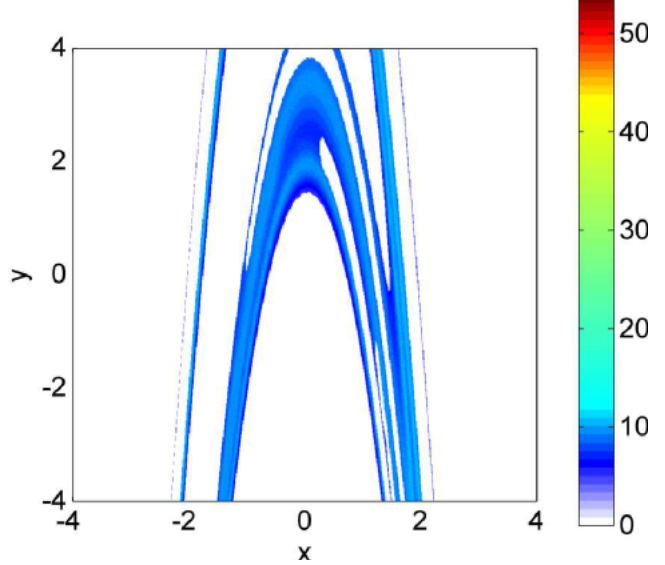

(b)

Notes: The figures (a) and (b) represent the square $x \in[-4,4]$ and $y \in[-4,4]$ in which we have computed the safe set. The figure (a) shows the average time escape of trajectories when no perturbation to escape is applied. In figure (b), the trajectories are induced to escape applying small control to go far away from the safe set. With this strategy, the average time escape is reduced about a factor 5.

\section{Conclusions}

We have proposed a new approach based on the partial control method, to handle transient chaotic dynamics affected by external disturbances. The goal of control is to keep trajectories chaotic during a desired time, and after that, to force the escape in the shortest possible time. To do that, we only have to compute the corresponding safe set which can be used in a dual way. In the attractive way, the perturbations are applied to put back the orbits in the safe set and therefore keep the chaotic behaviour. In the repulsive way, the perturbations drive the trajectories far away from the safe set to produce the escape. The control method was tested in the paradigmatic models, the 1D logistic map and the 2D Hénon map for a choice of parameters where the transient chaotic dynamics appears. In both cases, we show how to compute the safe set and how to use it to control the trajectories.

The main advantage of the method is the small amount of control necessary to apply, and the significant reduction of the time escape. In addition, we show that this approach is robust in the sense that is able to deal with noisy dynamics. The procedure to apply the method is also very simple, once the chaotic region is identified, and the upper values of the disturbance and control are specified, the computation of the safe set is automatic, making the method very easy to implement. 


\section{Acknowledgements}

The financial support from the Spanish Ministry of Economy and Competitiveness under Project No. FIS2013-40653-P is acknowledged.

\section{References}

Blanchini, F. (1999) 'Set invariance in control', Automatica, Vol. 35, No. 11, pp.1747-1767.

Capeáns, R., Sabuco, J. and Sanjuán, M.A.F. (2014) 'When less is more: partial control to avoid extinction of predators in an ecological model', Ecol. Complex, Vol. 19, pp.1-8.

In, V., Spano, M.L., Neff, J.D., Ditto, W.L., Daw, C.S., Edwards, K.D. and Nguyen, K. (1997) 'Maintenance of chaos in a computational model of thermal pulse combustor', Chaos, Vol. 7, No. 4, pp.605-613.

López, A.G., Sabuco, J., Seoane, J.M., Duarte, J., Januário, C. and Sanjuán, M.A.F. (2014) 'Avoiding healthy cells extinction in a cancer model', J. Theor. Biol., Vol. 349, pp.74-81.

Perc, M. and Marhl, M. (2006) 'Chaos in temporarily destabilized regular systems with the slow passage effect', Chaos Soliton Fract., Vol. 27, No. 2, pp.395-403.

Sabuco, J., Zambrano, S., Sanjuán, M.A.F. and Yorke, J.A. (2012a) 'Finding safety in partially controllable chaotic systems', Commun. Nonlinear Sci. Numer. Simulat., Vol. 17, No. 11, pp.4274-4280.

Sabuco, J., Zambrano, S., Sanjuán, M.A.F. and Yorke, J.A. (2012b) 'Dynamics of partial control', Chaos, Vol. 22, No. 4, p.047507.

Schwartz, I.B. and Triandaf, I. (1996) 'Sustaining chaos by using basin boundary saddles', Phys. Rev. Lett., Vol. 77, No. 23, pp.4740-4744.

Yang, W., Ding, M., Mandell, A.J. and Ott, E. (1995) 'Preserving chaos: control strategies to preserve complex dynamics with potential relevance to biological disorders', Phys. Rev. E, Vol. 51, No. 1, pp.102-110. 